\title{
Management of an accidental superior vena caval transection during a right pneumonectomy- A case report and assessment of different management options with review of literature
}

\author{
Kallol Dasbaksi*, Pinaki Majumdar, Siddharth Powah, Mohammad Zahid Hossain, Suranjan Haldar, Kaushik Mukherjee and Plaban Mukherjee \\ Department of Cardiothoracic Surgery, Medical College, Kolkata-700073, India
}

\begin{abstract}
We present a rare case of management of accidental transection of superior vena cava (SVC) during a right sided pneumonectomy for a tuberculous destroyed lung in an eighteen year old girl. While dissecting the right pulmonary artery (RPA) in a densely adherent and grossly distorted field for a planned right pneumonectomy, the SVC got transected resulting in torrential hemorrhage and severe hypotension. The ragged ends of the SVC were clamped. Fluids and inotropes were directly transfused into the right atrium (RA) which was exposed by opening the pericardium. Soon, the mean arterial pressure (MAP) could be stabilized around $50 \mathrm{~mm} \mathrm{Hg}$ which had dropped to $25 \mathrm{~mm} \mathrm{Hg}$. To protect the brain from effects of increased intra cranial venous hypertension (ICVH) due to SVC clamping, the head end of the table was elevated, injection thiopentone $(1000 \mathrm{mg})$ was given directly into RA and the head was wrapped with ice bags. Since the cut ends of the SVC was far apart and ragged, we could not bring them together for an end to end anastomosis. After heparinisation, a SVC to RA veno atrial (VA) shunt using venous cannulae was made and the SVC remained clamped for 20 minutes. With the establishment of the shunt, MAP increased to $80 \mathrm{~mm}$ Hg. Pneumonectomy was completed. Since no graft could be procured, the upper end of the SVC was anastomosed end to end with the cut end of the RPA and the lower end of SVC towards the RA was closed. Post operative recovery was uneventful and the girl is doing well 6 years after the procedure. Angiograms have shown a patent SVC to RPA anastomosis with a substantial retrograde flow through the intact azygos vein $(\mathrm{AzV})$.
\end{abstract}

\section{Introduction}

Injuries to superior vena cava (SVC) though rare, have been reported [1-4] during right sided pneumonectomy, some of which needed cross clamping of SVC for their successful repair. For patients whose SVC is non obstructed, its clamping may trigger a hemodynamic cascade of events. There is severe hypotension due to blood loss compounded by the absence of SVC return to the heart, which account for $35 \%$ of cardiac output in adults [5] and 50\% in children [6]. The reduced mean arterial pressure (MAP) results in reduction of cerebral perfusion pressure (CPP). Moreover, clamping the severed ends of SVC to arrest bleeding causes intra cerebral venous hypertension (ICVH) leading to increased intracranial pressure (ICP) and alteration of cerebral arteriovenous gradient. If obstruction remains unrelieved, it can lead to irreversible brain edema and intracerebral hemorrhage [7]. Safe duration of SVC and azygos vein (AzV) clamping has been claimed to be around one hour in experimental animals anesthetized with barbiturates [8], whereas in cases where ether was used, the animals died soon after such clamping [9]. So anesthetic management has definitive roles in prolonging safe duration of SVC clamping. The duration of SVC clamping can be increased if measures are taken to reduce the cerebral metabolic rate for oxygen (CMRO2) and central venous pressure (CVP) $[7,8,10]$. This report, an extended version of our earlier publication [11], outlines the management of accidental transection of a non obstructed SVC after clamping the cut ends, by (1) use of colloids, blood and inotropes directly into an exposed right atrium to combat hypotension, (2) intravenous thiopentone and maintenance of mild hypothermia for reducing $\mathrm{CMRO} 2$, and (3) creation of a temporary veno atrial (VA) shunt after heparinisation to reduce CVP and ICP, and (4) finally a cavo pulmonary anastomosis for drainage of the SVC after pneumonectomy. The role of azygos vein $(\mathrm{AzV})$ has also been observed as an important route of decompression of SVC during the period of clamping and also after cavo pulmonary anastomosis.

\section{Case report}

An eighteen year old female patient with a body weight $40 \mathrm{~kg}$, who had symptomatic destroyed right lung due to tuberculosis (Figure $1 \mathrm{~A}$ and $1 \mathrm{~B}$ ) was posted for right sided pneumonectomy. A $35 \mathrm{Fr}$ double lumen endobronchial tube (Mallinckrodt, MA, USA) was used and monitoring was done with ECG, pulse oxymetry, end tidal carbon di oxide (etCO2), temperature and intra arterial blood pressure. Central venous pressure (CVP) line was not used as was our practice during pneumonectomy. Maintenance of anesthesia was done with nitrous oxide/oxygen and isoflurane. Standard posterolateral thoracotomy through a $5^{\text {th }}$ space approach was done. There was thickened pleura

Correspondence to: Kallol Dasbaksi, Department of Cardiothoracic Surgery, Medical College, 88, College Street, Kolkata-700073, India, Tel: +919830321432; E-mail: kalloldb@yahoo.com

Key words: SVC transaction, intra cranial venous hypertension, glenn shunt, azygos vein

Received: April 06, 2015; Accepted: May 06, 2015; Published: May 08, 2015 


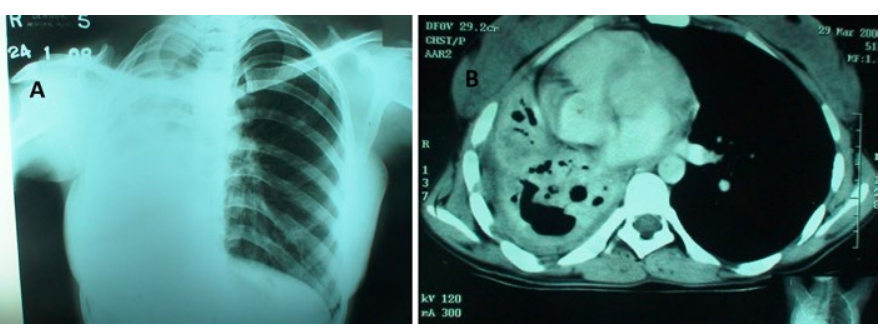

Figure 1. Pre operative chest X Ray (left) and CT (right) showing the destroyed right lung with mediastinal contents pulled into the right chest cavity due to fibrotic lung.
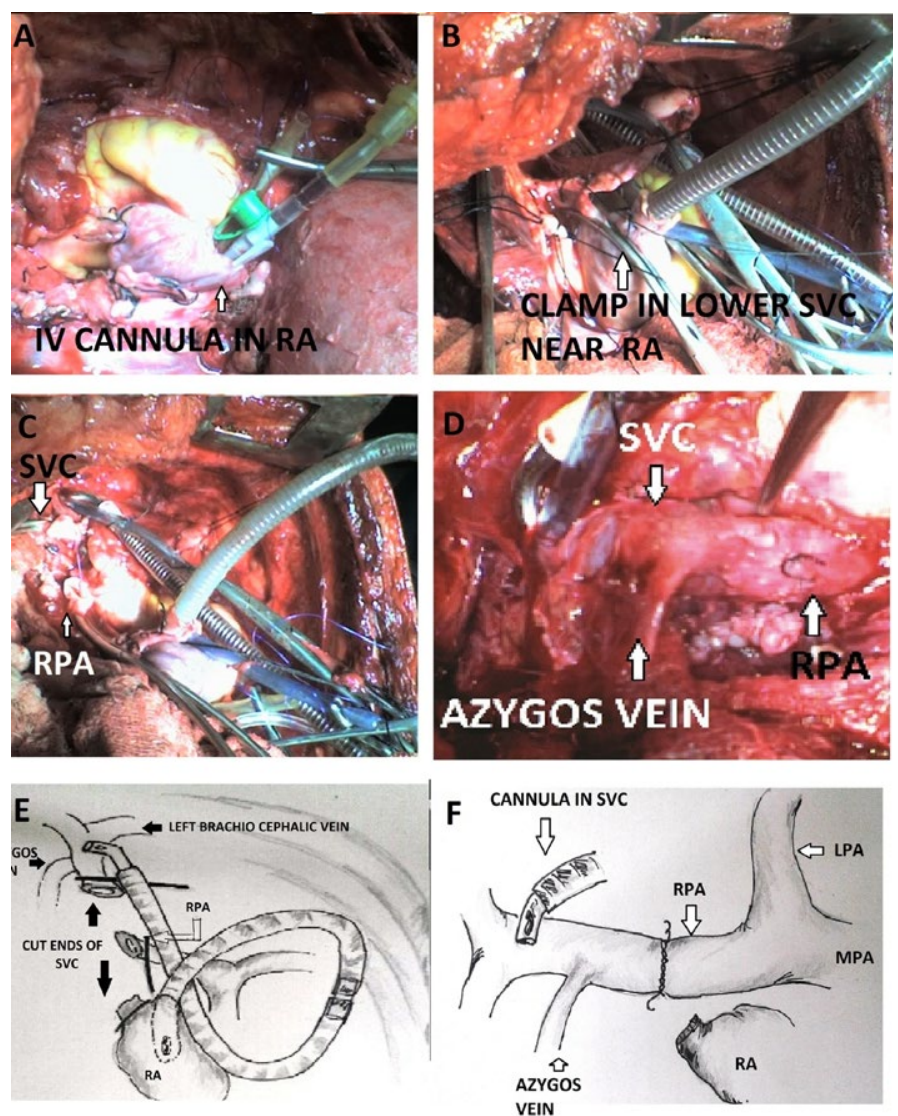

Figure 2. Intra operative photographs taken in the apical region of right thorax as viewed in right lateral thoracotomy showing (A) a $18 \mathrm{G}$ intravenous cannula inserted within the right atrium and secured with a purse string. (B) Vascular clamps applied to the severed lower end of SVC shown by an arrow. (C) The upper stump of SVC (upper arrow). Both B and $\mathrm{C}$ show cannula in right atrial appendage and upper end of SVC better shown in C. The lower stump adjacent to the right atrium (lower arrow) is already sutured and declamped. The lower arrow points towards the stump of the right pulmonary artery (RPA). A right angled Pacifico cannula inserted into the upper stump and a straight cannula into the right atrial appendage is visible. (D) Shows completed end to end anastomosis between the upper stump of SVC and right pulmonary artery (white arrow head) before removal of the SVC cannula. (E) and (F) are the schematic diagrams and self explanatory. (C,D,E, and F are redrawn from Dasbaksi et al. [11], JTCVS,2014 with permission).

with gross adhesions and fibro-cavitory lesions of the underlying destroyed lung. While dissecting the RPA, the SVC was accidentally injured and subsequently got transected by repeated application of vascular clamps while trying to control the massive blood loss. There was severe hypotension, with the MAP falling to $25 \mathrm{~mm} \mathrm{Hg}$ and the etCO2 was $8-9 \mathrm{~mm} \mathrm{Hg}$. Vascular clamps could finally be applied to the divided ends of the SVC and bleeding was controlled (Figure 2B, $2 \mathrm{C}$ ). The pericardium was opened and direct access was secured to the right atrium (RA) by a $18 \mathrm{G}$ intravenanous cannula (Figure $2 \mathrm{~A}$ ).
One litre of Ringer Lactate, and $500 \mathrm{ml}$ of Gelofusine (succinylated gelatin), 2 units of whole blood were infused. Inotropic infusion with dopamine and adrenaline was started. MAP increased to $50 \mathrm{~mm} \mathrm{Hg}$ with these measures. To counter-act the presumed rise in ICP due to SVC clamping, $1000 \mathrm{mg}$ intravenous thiopentone was administered into the RA line. Ice packs were wrapped around the head. The patient was hyperventilated. The etCO2 was maintained around $30 \mathrm{~mm} \mathrm{Hg}$. Oro pharyngeal temperature was recorded to be 34 degree centigrade (C). In the mean time a temporary SVC to RA shunt was then made after heparinisation (using unfractionated heparin at the dose of $2 \mathrm{mg}$ per kg body weight) using a $20 \mathrm{~F}$ right angled cannula for SVC and $28 \mathrm{~F}$ straight cannula for the RA appendage (Figure 2B, 2C, 2D) connected with a straight connector. The SVC remained clamped for 20 minutes while constructing the SVC to RA shunt. During this period fluid was cautiously infused, with assessment of pulmonary artery tension by digital palpation over the exposed main pulmonary artery to avoid fluid overloading. Blood gas and acid base parameters were attempted to be kept near normal values, normoglycemia was maintained throughout the clamp period and thereafter. When the shunt started functioning, MAP increased to $80 \mathrm{~mm} \mathrm{Hg}$ and urine output became normal after an initial period of oliguria. Pneumonectomy was then completed which required almost 120 minutes for completion. Since no grafts were available, the operation was completed by anastomosing the cut end of the SVC to the cut end of the RPA (Figure 2D and 2F) using 6-0 polypropylene. The lower end of the SVC near the RA was closed with 5-0 polypropylene. The AzV draining into the upper stump of SVC was left intact. Protamine was given, decannulation was done and the chest was closed in usual manner with single intercostal drain. Extubation could be done in the operation room after the patient was warmed and became hemodynamically stable. There was no cognitive dysfunction. However, puffiness of the face (Figure 3) persisted for one week post operatively but diminished slowly thereafter. Recovery was uneventful and she could be discharged with oral aspirin $75 \mathrm{mg}$ daily. She was then lost to followup but revisited our out patient department after 4 years for review of the thoracotomy scar (Figure 4) which was found to be healthy. We admitted her for investigations for cardio respiratory function. She had discontinued aspirin soon after discharge and did not have any respiratory problems and was in NYHA class 1 . A chest roentgenogram and computed tomogram $(\mathrm{CT})$ was then done (Figure $5 \mathrm{~A}, 5 \mathrm{~B}$ ) which showed hyperinflated left lung and a right chest opacity as is normal after right sided pneumonectomy. Saturation was $98 \%$ in

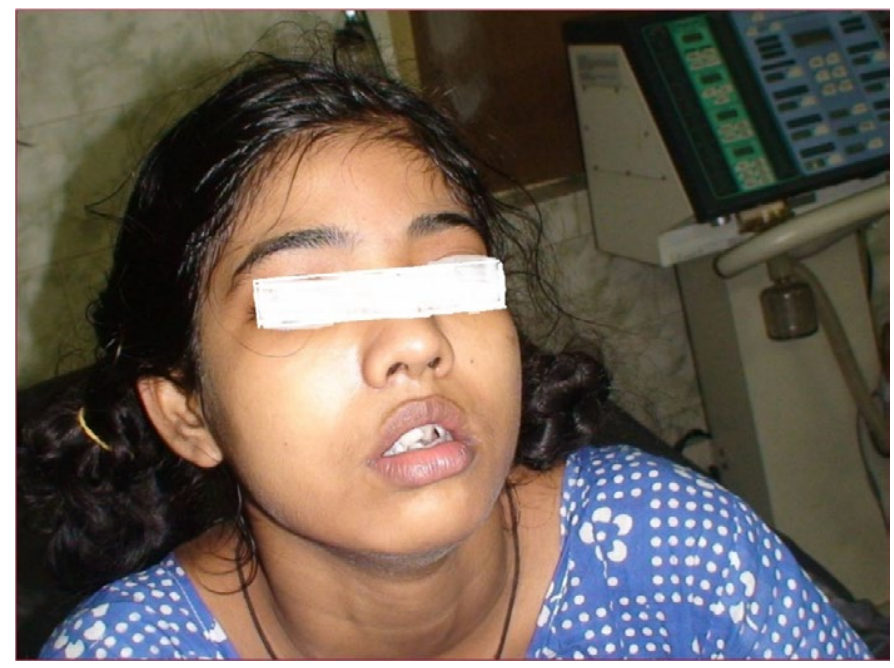

Figure 3. Post operative picture of the patient showing slight puffiness of face. 


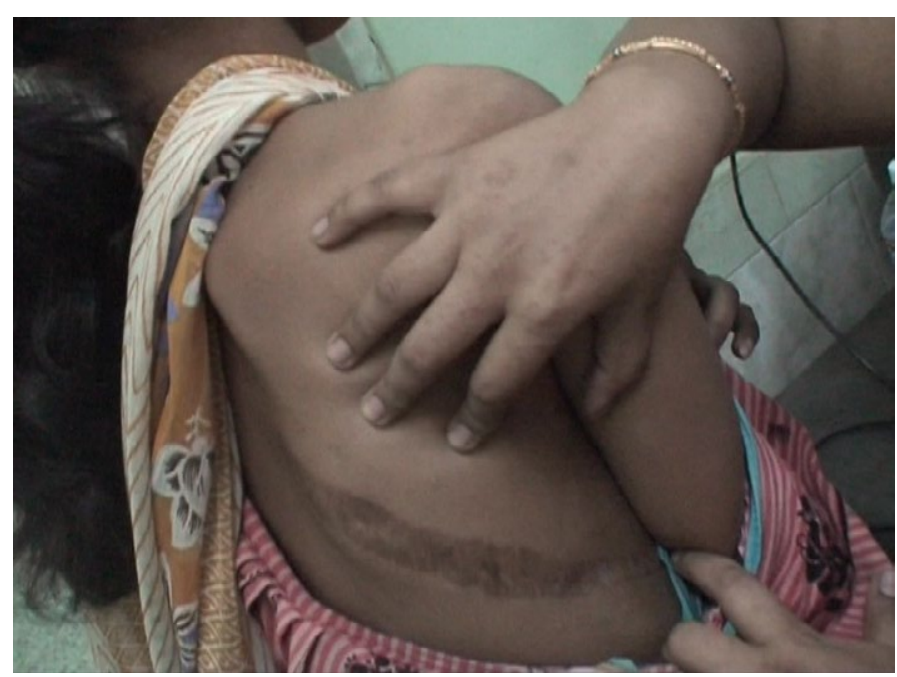

Figure 4. Shows the posteolateral scar in the right side in the fourth post operative year.

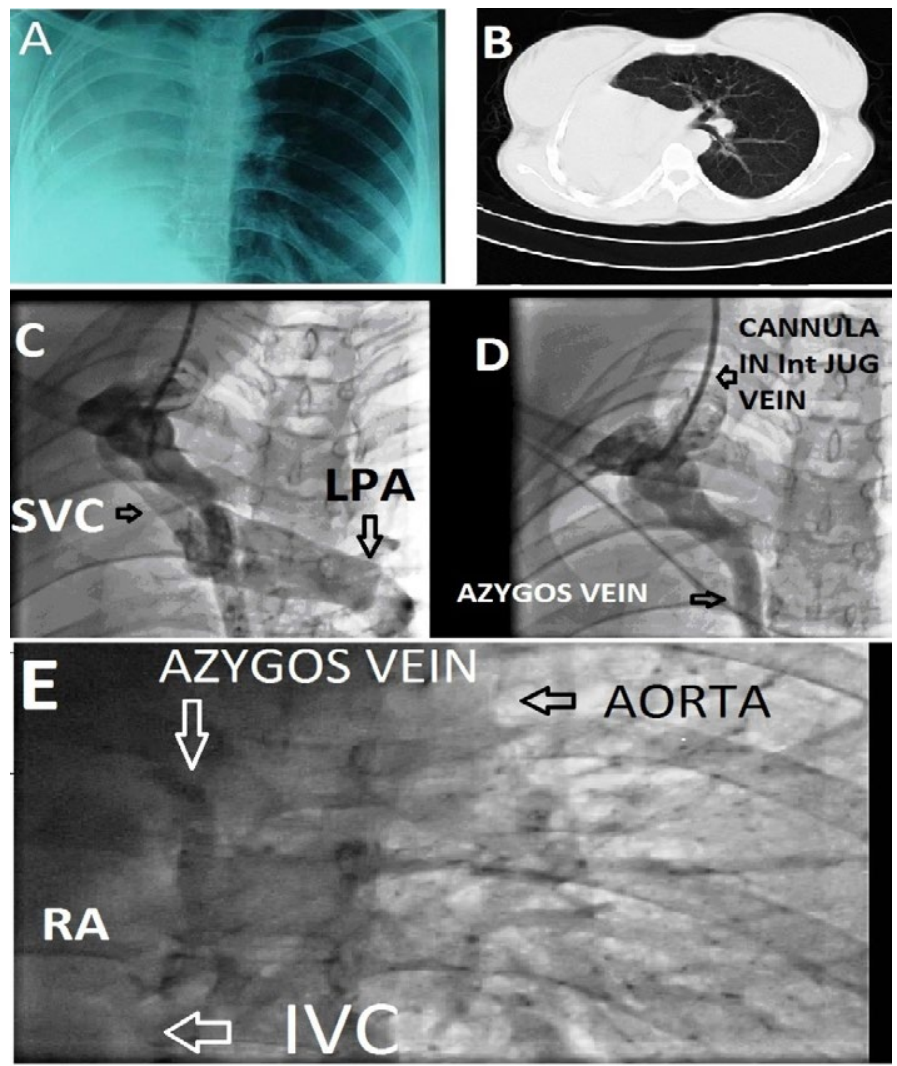

Figure 5. Post operative photographs on the $4^{\text {th }}$ year: (A) chest X Ray showing opacity in the right hemithorax with dextroposed heart. (B) Showing the CT scan confirming the findings of Chest X Ray with hyperinflated left lung. (C) Shows passage of radio opaque dye on right internal jugular venous angiography into both left pulmonary artery (lower arrow head) and the azygous vein. Upper arrow shows the outline of the right internal jugular vein. (D) Shows further passage of dye through the azygous vein (horizontal arrow) after the flow through the left pulmonary artery stopped. (E) shows mild opacification of the right atrium at a later phase due to retrograde passage of dye through the azygos vein. (redrawn from Dasbaksi et al. [12], JTCVS, 2014 with permission)

room air. Echocardiography and doppler study showed flow from SVC to RPA and cardiac function was normal. Cardiac catheterisation was done. It showed the pulmonary artery pressure to be $20 / 10 \mathrm{~mm} \mathrm{Hg}$ (mean $13 \mathrm{~mm}$ ). SVC was separately cannulated through the internal jugular vein and the CVP was found to have a mean value of $14 \mathrm{~mm}$ $\mathrm{Hg}$. SVC angiography showed dye going to the left PA but majority going into the $\mathrm{AzV}$ (Figure 5C, 5D and 5E) and was visible entering right atrium through inferior vena cava at a later phase. IVC and SVC angiographic video is also available.

\section{Discussion}

\section{Problems faced with SVC clamping}

An obstructed SVC may be clamped for vascular resection reconstruction while removing an invasive tumor like thymoma or lung carcinoma [12] without much hypotension as there is adequate return though venous collaterals which develop with these conditions. Clamping an unobstructed SVC can result in sharp increase of CVP leading to ICVH and raised ICP because the venous collateral channels (as described below) are not adequate in these cases. MAP is reduced due to loss of SVC return. Concomitantly, CPP is also reduced with risk of brain ischemia since $\mathrm{CPP}=\mathrm{MAP}-\mathrm{ICP}$ or $\mathrm{CVP}$, as CVP is only slightly more or less equal to ICP [13]. If the clamp time is short (4 to 10 minutes), CPP can be maintained by raising the MAP by judicious use of fluids and inotropes [14]. However, if the clamp time is prolonged, a raised ICP fails to decrease after release of SVC clamp despite reduction of CVP. This is caused by no-reflow phenomena because of sufficient structural damage of the microvasculature of brain, leading to brain edema, hypoxia and intra cerebral metabolic acidosis [7] unless measures to reduce CMRO2 are taken. Clamping a patent SVC without collaterals due to accidental SVC injury during pneumonectomy presents an even greater exigency due to difficulty to arrange for a quick logistic support, even to an experienced cardiothoracic surgeon and anesthetic team not contemplating such an event, because hypotension and shock due to severe blood loss within minutes, coupled with low CPP and raised ICP, ensues rapidly [1-4]. The following strategies can help as a solution in prolonging the duration of clamping during such an event with successful outcome.

\section{Anesthetic management}

These include (i) maintenance of normal MAP, (ii) adopting measures to maintain cellular integrity of brain by decreasing the brain metabolism by pharmacological agents which includes drugs as well as inhalational anesthetic gases, and (iii) hypothermia. Fall in MAP was corrected in our case by judicious use of crystalloids/ colloid and vasopressors directly into the exposed RA as venous access in the upper extremity became useless and creating an intravenous access in the collapsed veins of lower limb in lateral position was very difficult. Although blood gas could not be corrected adequately during the period of clamping, it became normal after the VA shunt started functioning. Other measures adopted were maintenance of normoglycemia during clamping. $\mathrm{CMRO} 2$ was attempted to be reduced by a bolus dose of $1000 \mathrm{mg}$ thiopentone and cooling of head by ice packs. Due to cool operation room temperature and open thoracotomy wound, nasopharyngeal temperature had dropped to 34 degree $\mathrm{C}$ and was maintained around these figures by regulation of ambient temperature. By following these measures, 20 minutes of clamping was tolerated before completion of VA shunt. In cases where continuous electro encephalogram (EEG) is monitored [2], studies have shown, that intravenous barbiturates decrease cerebral metabolism in a dose dependent manner. When EEG becomes isoelectric, $\mathrm{CMRO} 2$ is reduced by $50 \%$ and additional doses of barbiturates after flattening of EEG provide no additional metabolic depression [10]. In experimental animals, SVC and AzV ligation has resulted in death within minutes 
where ether was used as anesthetic agent [9], whereas 1 hour of similar clamping was tolerated when anesthesia was maintained by intravenous barbiturates [8]. But, in animals induced with barbiturates and maintained with isoflurane, cerebral edema and hemorrhage were observed after 35 minutes [7] where barbiturates were not used during clamping. Observations regarding usefulness of barbiturates have also been made in similar clinical [2] case reports. However, recent review has failed to demonstrate the neuroprotective action of thiopentone ubiquitously [15]. Mild hypothermia of 34 degree $\mathrm{C}$ also added towards brain protection. Baughman (2002) observed that for every degree $\mathrm{C}$ decrease in temperature, cerebral metabolism is reduced by $5-7 \%$ and a reduction in temperature from 37 to 34 degree $\mathrm{C}$ produces a $15-20 \%$ reduction in cerebral metabolism, which is far less than the $50 \%$ decrease seen with flattening of EEGs. Therefore, hypothermia's protective effect is not mediated solely by metabolic depression. Several mechanisms have been proposed, which include suppression of glutamate release, reduced calcium influx and gamma-aminobutyric acid release, and reduction of nitric oxide production which is involved in producing oxygen free radicals [10].

\section{Maintaining the Azygos flow}

Azygos vein flow came to prominence because of "azygos flow principle" or low flow state. According to this principle, little antegrade flow of 8 to $10 \mathrm{cc} / \mathrm{kg}$ through a patent $\mathrm{AzV}$ available for filling the right atrium, after total venous occlusion in anesthetized dogs is compatible with life for 30 to 35 minutes. The procedure involved clamping of SVC cranial to its junction with $\mathrm{AzV}$ and the inferior vena cava caudally, only allowing $\mathrm{AzV}$ inflow into the RA. This was the foundation of effective controlled cross circulation for short periods of cardiac bypass by Lillehei et al. using less flow, in contrary to high flow, during cardiopulmonary bypass, which was prevalent in early 1950s [16]. But it was retrograde flow through the $\mathrm{AzV}$ which was found to sustain adequate circulation after ligation of SVC caudal to its junction with $\mathrm{AzV}$, in dogs by Carlson in 1934, all of which survived after a few days of listlessness. But ligation of SVC cephalad to the AzV-SVC drainage in dogs resulted in death of all the animals [9] within a few hours. This experiment proves the usefulness of $\mathrm{AzV}$ as the principal collateral channel in acute occlusion of SVC, because the other two collateral routes i.e. vertebral veins and internal thoracic veins, were already patent in both the groups of animals. The fourth one, the external thoracic vein, which is the superficial collateral system from the subclavian and axillary vein to femoral vein [17] becomes prominent in chronic occlusion of SVC. Importance of AzV has also been proved in subsequent experimental study of Gonzalez-Fazardo et al. (1994) who showed an abrupt increase of CVP from 7 to $17 \mathrm{mmHg}$ on clamping of SVC in dogs [7]. On further clamping of AzV after 5 minutes, there was a sharp increase in CVP to $32 \mathrm{~mm} \mathrm{Hg}$, which explains that some degree of reversal of flow through the AzV had been established before it was clamped. This advantage has been utilized by surgeons during construction of off pump bi directional Glenn shunt in children having single ventricle physiology, without use of any VA shunt [14]. Successive intermittent clamping of SVC caudal to the AzV junction, before final clamping and transection of SVC, allowed the CVP to settle down towards a lower value after an initial rise. AzV was ligated only after declamping the SVC on completion of BDG to allow increased flow through the shunt. Francesco Leo et al. [12] reported haemodynamic instability during SVC cross clamping for venous reconstruction in six of their patients who had either a blocked $\mathrm{AzV}$ or a patent $\mathrm{AzV}$ clamped during operation. Release of AzV clamp restored stability. Retrograde flow through the $\mathrm{AzV}$ also explains tolerance of 20 minutes of SVC clamping in addition to fluids and inotropes in our patient, and also in other cases of such accidental injuries [1,2]. Accidental ligation of SVC during pneumonectomy for tuberculosis has also been reported to be tolerated for a few hours before being noticed and reoperated for SVC reconstruction [18]. Modern anesthesia has allowed both SVC and $\mathrm{AzV}$ occlusion to be tolerated only upto 45 minutes in clinical practice as longer period of clamping can produce brain damage specially when $\mathrm{AzV}$ is ligated [17], though there are anecdotal reports of 115 minutes of successful SVC and AzV clamping during reconstruction [19]. A VA shunt would be a safe option during prolonged clamping.

\section{Decompression of a clamped SVC by creation of a tem- porary VA shunt}

This is helpful if there is haemodynamic instability despite fluid and inotropic support on clamping a non obstructed SVC or in situations where clamping for considerable period is required for completion of associated surgical procedures [1,7]. In our case VA shunt was maintained for 2 hours for completion of pneumonectomy.

Surgical considearions for SVC reconstruction: Choices for SVC reconstruction by replacement includes autogenous superficial femoral vein (SFV) and spiral vein graft, gluteraldehyde treated autologous and bovine pericardial grafts, bovine internal jugular vein graft and aortic homograft [18]. In cases of partial SVC injury endovascular grafts [4] may also be used. Polytetrafluoroethylene (PTFE) grafts [12] which are available in a variety of sizes would have been the ideal choice, but could not be procured during surgery in our case. In order to provide an unobstructed SVC drainage we had created a SVC to RPA end to end cavo pulmonary (Glenn) shunt as a rescue procedure and has been described in our earlier surgical case report [11]. In similar situation, cavo atrial anastomosis has also been reported [3] but the $\mathrm{AzV}$ division during this anastomosis for gaining length of SVC was too risky in the absence of a VA shunt.

Function of the cavo pulmonary shunt after 4 years: Echocardiography done 4 years following the operation showed normal flow through the SVC-RPA anastomosis. Pulmonary arterial pressure was measured to be $20 / 12 \mathrm{~mm} \mathrm{Hg}$ with a mean value of $13 \mathrm{mmHg}$ on cardiac catheterisation. On SVC cannulation a mean CVP of $14 \mathrm{~mm}$ $\mathrm{Hg}$ was found which is in the slightly higher range, and angiography showed not only flow through the left PA but considerable retrograde flow through the AzV which did not allow the CVP to rise higher. There was no radiological evidence of any intra pulmonary vascular malformations as sometimes observed in cases of Glenn shunt and Fontan operation [20] since the remaining left lung is being perfused by inferior vena caval flow carrying the hepatic factor.

\section{Conclusion}

Clamping an unobstructed SVC may have to be done in unavoidable circumstances like SVC injury to control bleeding. Since the cascade of abnormal haemodynamics leading to reduced CPP starts developing with clamping, steps must be taken to combat hypotension and reduction of brain metabolism. Patency of an intact $\mathrm{AzV}$ is to be maintained till completion of SVC reconstruction. Establishment of a temporary SVC to RA shunt after heparinisation is a safe option before repairing the injured SVC. We have used CVP in every case of pneumonectomy/ lobectomy since this event. In addition to its original objectives, the presence of a CVP cannula in the SVC can be a guide to avoid injury during right sided dissection. 
Dasbaksi K (2015) Management of an accidental superior vena caval transection during a right pneumonectomy-A case report and assessment of different management options with review of literature

\section{References}

1. Agaskar R, Goswami J, Bartarya S (2008) Successful Resuscitation in Accidental Complete Transection of Superior Vena Cava during Right Pleuropneumonectomy. Ind J Anaesth 52: 569.

2. Vretzakis G, Didilis V, Dragoumanis C, Mikroulis D, Lazarides M (2006) Nonprogrammed clamping of superior vena cava. The anesthesiologist's Achilles' heel. Acta Anaesthesiol Belg 57: 59-62. [Crossref]

3. Van Natta TL, Parekh KR, Dearmond DT, Iannettoni MD (2010) Damage control: cavoatrial anastomosis during a catastrophic right intrapericardial pneumonectomy. Tex Heart Inst J 37: 587-590. [Crossref]

4. Anaya-Ayala JE, Charlton-Ouw KM, Kaiser CL, Peden EK (2009) Successful emergency endovascular treatment for superior vena cava injury. Ann Vasc Surg 23: 139-141. [Crossref]

5. Mohiaddin RH, Wann SL, Underwood R, Firmin DN, Rees S et al (1991) Vena caval flow: assessment with cine MR velocity mapping. Radiology 178: 1229-1235. [Crossref]

6. Salim MA, DiSessa TG, Arheart KL, Alpert BS (1995) Contribution of superior vena caval flow to total cardiac output in children. A Doppler echocardiographic study. Circulation 92: 1860-1865. [Crossref]

7. Gonzalez-Fajardo JA, Garcia-Yuste M, Florez S, Ramos G, Alvarez T, et al. (1994) Hemodynamic and cerebral repercussions arising from surgical interruption of the superior vena cava. Experimental model. J Thorac Cardiovasc Surg 107: 1044-1049. [Crossref]

8. Masuda H, Ogata T, Kikuchi K (1989) Physiological changes during temporary occlusion of the superior vena cava in cynomolgus monkeys. Ann Thorac Surg 47: 890-896. [Crossref]

9. Carlson HA (1934) Obstruction of superior vena cava - An experimental study. Arch Surg 29: 669-677.
10. Baughman VL (2002) Brain protection during neurosurgery. Anesthesiol Clin North America 20: 315-327. [Crossref]

11. Dasbaksi K, Mukherjee K, Saha E, Mukherjee P (2014) Salvaging an accidental superior vena caval transection during a right pneumonectomy by creating a Glenn shunt: a case report and review of the literature. J Thorac Cardiovasc Surg 148: e147149. [Crossref]

12. Leo F, Grazia LD, Tullii M, Gasparri R, Borri A, et al. (2007) Hemodynamic instability during superior vena cava crossclamping: Predictors, management, and clinical consequences. J Thorac Cardiovasc Surg 133: 1105-1106. [Crossref]

13. Mishra LD (2002) Cerebral blood flow and anaesthesia: A Review, Indian J. Anaesth 46: 87-95.

14. Bhan A (2008) Off-pump bi-directional Glenn shunt: How I do it? Ann Pediatr Cardiol 1: 131-134. [Crossref]

15. Bilotta F, Gelb AW, Stazi E, Titi L, Paoloni FP, et al. (2013) Pharmacologica perioperative brain neuroprotection: a qualitative review of randomized clinical trials. Br J Anaesth 110 Suppl 1: i113-120. [Crossref]

16. Loomis Bell AL Jr, Fitzpatrick HF, Andreae E, Shimomura S, Pingeon R (1958) The influence of the rate of perfusion on physiologic responses during cardiac bypass. Ann Surg 148: 968-978. [Crossref]

17. Macchiarini P, Dartavelle P (2005) The use of prosthetic grafs for replacement of Superior vena cava, Chapter 172, in General Thoracic Surgery, (6th Edn.), In: Shields Th. W, LoCicero J, Ponn R. B, RuschV. W, (Eds), Publisher: Lippincott Wiliams and Wilkins: 2572-2573.

18. Ohri SK, Lawrence DR, Townsend ER (1997) Homograft as a conduit for superior vena cava syndrome. Ann Thorac Surg 64: 531-533. [Crossref]

19. Inoue H, Shohtsu A, Koide S, Ogawa J, Inoue H (1990) Resection of the superior vena cava for primary lung cancer: 5 years' survival. Ann Thorac Surg 50: 661-662. [Crossref]

20. Kopf GS1, Laks H, Stansel HC, Hellenbrand WE, Kleinman CS, et al. (1990) Thirtyyear follow-up of superior vena cava-pulmonary artery (Glenn) shunts. J Thorac Cardiovasc Surg 100: 662-670. [Crossref]

Copyright: (C2015 Dasbaksi K. This is an open-access article distributed under the terms of the Creative Commons Attribution License, which permits unrestricted use, distribution, and reproduction in any medium, provided the original author and source are credited. 